\title{
Syntheses Approach of 2-D Oxide Family of Graphene for Supercapacitor Application (A-Review)
}

\author{
PARIMAL ROUTH \\ Department of Chemistry, Charuchandra College, 22, Lake Road, Kolkata-700029, India. \\ *Corresponding author E-mail: parimalpsu@gmail.com \\ http://dx.doi.org/10.13005/ojc/360603
}

(Received: September 12, 2020; Accepted: November 20, 2020)

\begin{abstract}
More recently, 2-D graphene oxide (GO)/reduced graphene ( $\mathrm{GGO}$ ) have altered the direction of modern science with material chemistry and physics by research as they offer different key advantages. These are (i) atomically thin 2-D nanosheets (NSs) provide a large surface area (ii) presence of maximum chemically reactive sites, and (iii) higher mechanical strength and flexibility. Considering the progress of graphene research, we broadly and crucially discuss the formation of the growing family of 2-D GO/rGO in this review paper. Synthesis methodologies are compared, focusing to offer signs for emerging novel and adaptable synthetic methods. Their advantage use in the fields of supercapacitor are highlighted in this review.
\end{abstract}

Keywords: Graphene oxide, Reduced graphene oxide, Two-dimensional, Oxidation, Reduction.

\section{INTRODUCTION}

In past few years, we have observed massive development in two-dimensional (2-D) materials research. 2-D materials have been shifting the scenery of many areas in science, mainly energy and biomedical field. Terrific work are continuing to discover the entire science of 2-D materials. 2-D materials are solid crystals having large lateral dimension ( 100 nm-1,000 nm) compare to their thickness $(\sim 1 \mathrm{~nm})$. The atomic thick 2-D layers are formed by strong intralayer covalent bonding among different atoms and the 2-D layers are interconnected by weaker interlayer Van der Waals to form corresponding bulk crystals. ${ }^{1,2}$ Based on the chemical constituents 2-D materials are generally categorized as pristine graphene, hexagonal boron nitrides, transition metal di-chalcogenides (TMDCs), 2-D oxide materials and Mxenes etc. The elemental zoo of 2-D materials is shown in Fig. 1. In this elemental zoo different types of 2-D materials are highlighted by different symbols. Mainly, the elements C, B, P, Si, Sn, Ge, Bi form 2-D allotropes. Generally, 'ene' suffix is used in their names. For example, graphene, phosphorene, borophene, germanene, silicene, etc. ${ }^{3-8}$

Graphene oxide/Reduced graphene is most valuable 2-D oxide material in the area of science and technology. Some important characteristic of 2-D GO/rGO are (i) their unique two dimensional structure permits large effective surface area which enhances catalytic activities. (ii) Presence of their intrinsically redox active oxygenated groups

This is an Open Access article licensed under a Creative Commons license: Attribution 4.0 International (CC- BY). Published by Oriental Scientific Publishing Company @ 2018

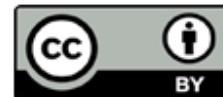


enhancements their electrochemical activity. For example, as an electrode material of supercapacitor, it store electrical charge in pseudocapacitive way. (iii) Their high mechanical strength and flexibility help to use them in different flexible, portable electronic devices. (iii) Good electrical conductivity of rGO which helping to use electrode material. The presence of different physical, chemical, and exotic electronic properties of GO/rGO have created a multipurpose opportunity for applications in sensors, surface-enhanced Raman spectroscopy (SERS), lubricants, field-emission devices, photovoltaic devices, transparent conducting electrodes, supercapacitors, fuel cells, lithium-ion batteries and photocatalysis. ${ }^{9-14}$ The multidimensional properties and applications of GO and rGO, hence currently researchers concentrated tremendous attention on synthesis because to find of high quality bulk amount $\mathrm{GO} / \mathrm{rGO}$ materials. Regarding these point of issues I have discussed in this review article about the various synthesis approach of $\mathrm{GO} / \mathrm{rGO}$ with their limitation and advantages.

Supercapacitor is such an energy storage device capable to store high energy density that bridge between high energy dense battery and ultrahigh power dense conventional capacitor. Further its light weight, flexibility with long cyclic stability has facilitated its commercialization. The supercapacitor have ability for supplying reasonable amount of energy due to ultrahigh power, it is useful during peak power demands where all the energy storage devices become abortive. These distinct properties of supercapacitors permit to be applied as prompt starting or reformative slowing in vehicles, and industrial equipment in which all energy-storage devices become failed. ${ }^{15-17}$ Basically conventional supercapacitors contain of two electrodes separated via an ion-permeable membrane and whole system i.e. electrode and separator are deep in electrolyte solution. Both electrolyte solution and electrode are very vital for supercapacitor as charge is stored by interaction of electrode and electrolyte. Electrodes are considered as heart of the supercapacitor as they have the foremost role on charge storing. Mainly different form of graphene, reactive carbon, oxides of transition metal, metal dichalcogenides, and some conducting macromolecules (polyaniline, polypyrrole and 2-D polymer) are utilized for electrode materials. Depending upon the nature of the electrode materials, acidic electrolytes
$\left(\mathrm{H}_{2} \mathrm{SO}_{4}, \mathrm{H}_{3} \mathrm{PO}_{4}\right.$, etc.), basic electrolytes $(\mathrm{KOH}$, $\mathrm{LiOH}$, etc.), neutral electrolytes ( $\mathrm{Na}_{2} \mathrm{SO}_{4}$, $\mathrm{LiCl}$, etc.) are used either in aqueous state or in gel state (polyvinyl alcohol based) for aqueous and solid state supercapacitor, respectively. Herein, we focus the discussion on 2-D GO and rGO and aim to cover the latest developments of different synthesis routes and highlight its unique supercapacitor application. ${ }^{12,18}$

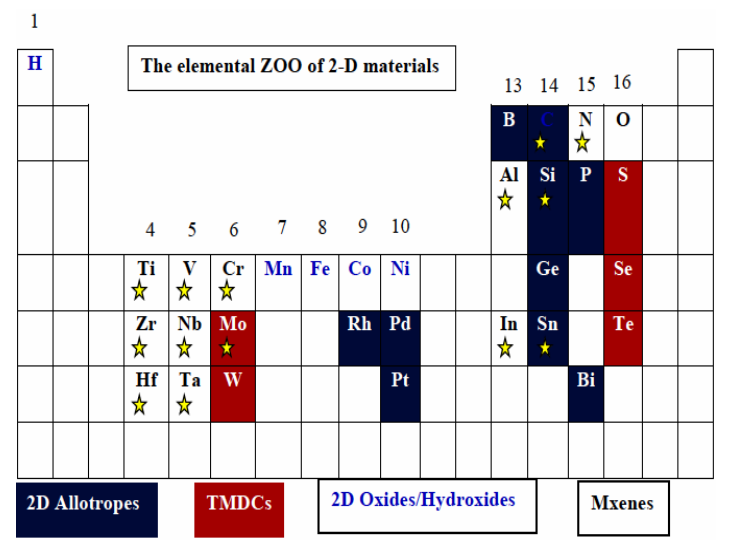

Fig. 1. The elementary zoo of 2-D materials. In this zoo different types of 2-D materials are highlighted by various symbols

\section{Synthesis of 2-D GO and rGO}

Graphene oxide and reduced graphene are very beneficial carbonaceous 2-D oxide materials. $\mathrm{GO}$ is prepared by exfoliation and oxidation of graphite. ${ }^{19}$ Actually, it is the oxidised form of graphene. Where, graphene is the mono layer of $\mathrm{sp}^{2}$ hybridised carbon organised as hexagonal honeycomb framework. Because of its regular $\pi$ conjugation of $\mathrm{sp}^{2}$ carbon, it is a zero bandgap semimetal. The GO contain various oxygenated groups as epoxy, carboxyl, hydroxyl, carbonyl etc. are incorporated in the graphene sheet. The incorporation of oxygenated groups generates $\mathrm{sp}^{3}$ carbons in plane of the sheet which breaks the conjugation and convert it to insulator and become not suitable for electrical application. So, to regain its conjugation it is reduced. Mainly the epoxy and hydroxyl groups of its plane are reduced and the oxygenated groups $(-\mathrm{COOH},>\mathrm{C}=\mathrm{O},-\mathrm{OH})$ of edges remain intact. This is termed as $\mathrm{rGO}$. The structure of graphene, GO and rGO are shown below in Figure 2. ${ }^{14,20,21}$

\section{Synthesis of Reduced Graphene Oxide}

The handiest route to yield rGO is exfoliationoxidation of graphite layer to GO, followed by its reduction to rGO as represented below in Figure 3. 

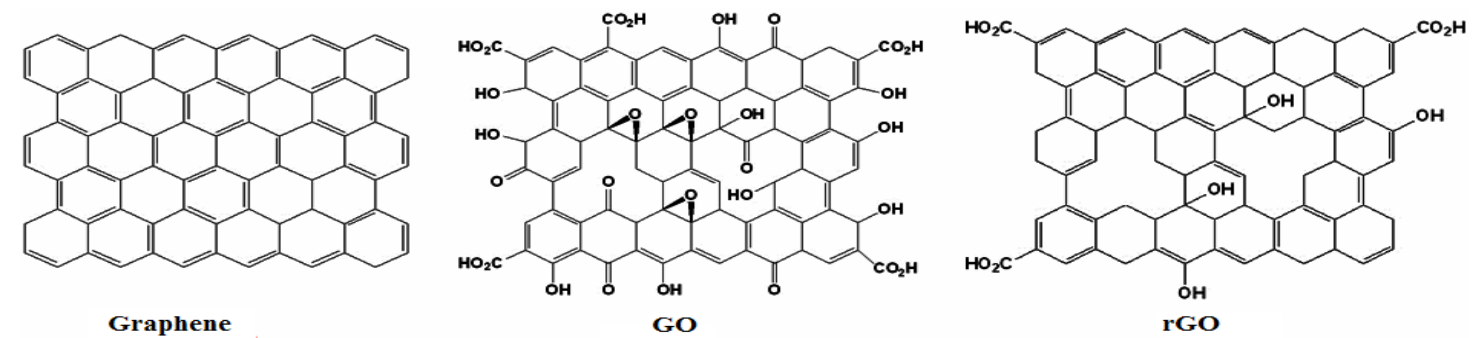

Fig. 2. Structure of Graphene, GO and rGO

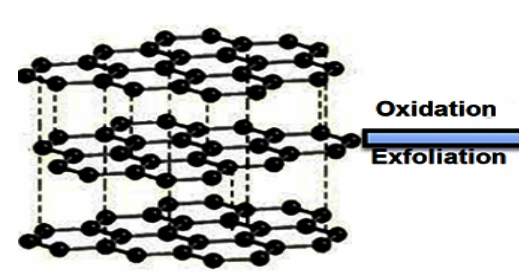

Graphite

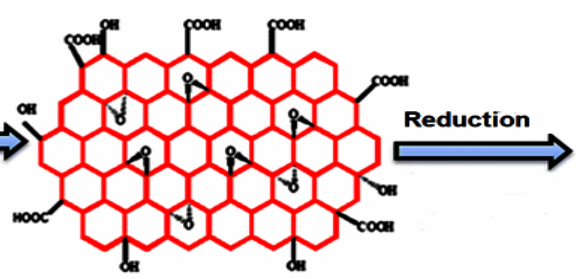

GO

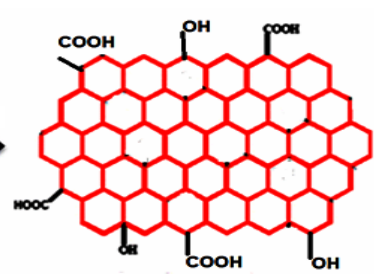

rGO

Fig. 3. Schematic representation of synthesis route of rGO (Ref. $\left.{ }^{21}(\mathbf{b})\right)$

\section{Synthesis of Graphene Oxide}

To improve the solubility of graphite we need further convert to $\mathrm{GO}$ due to more practical application in various field. The bulk form of GO, conventionally named is graphitic oxide or graphitic acid. In 1855 Brodie was first synthesized GO via constant treatment of Ceylon graphite with $\mathrm{KClO}_{3}$ and fuming $\mathrm{HNO}_{3}{ }^{22}$ After that, various methods were established for preparation of graphitic oxide, but all these methods were mainly use of strong oxidising mixture having different strong acids and oxidizing reagents. ${ }^{23}$ But in large scale, GO was first synthesized by Hummers and Offeman in 1957 and this famous method is known as 'Hummers' Method'. ${ }^{24}$ In this synthesis graphite is oxidized in 3 weight equivalent $\mathrm{KMnO}_{4}$ with 0.5 weight equivalent $\mathrm{NaNO}_{3}$ in concentrated $\mathrm{H}_{2} \mathrm{SO}_{4}$. To improve the oxidation of graphite, 6 weight equivalent $\mathrm{KMnO}_{4}$ is used instead of 3 weight equivalent and this method is termed as 'Modified Hummers' Method'. Recently, Tour group have established 'Improved Method', where graphite is oxidised by its 6 weight equivalent $\mathrm{KMnO}_{4}$ in 9:1 $\mathrm{H}_{2} \mathrm{SO}_{4}$ and $\mathrm{H}_{3} \mathrm{PO}_{4}$ mixture to avoid the formation of toxic NOx gas. ${ }^{25}$

\section{Reduction of Graphene Oxide}

Graphene Oxide contains lots of oxygen holding groups which improved the solubility of $G O$ in wide range of different polar and non-polar solvent. GO has localized $\pi$ electrons due to presence of epoxy linkage and defect which hinder the conductivity of GO sheets. Thus we need further control reduction of GO sheet to improve the conductivity and preserve its solubility. Actually the percentage of oxygenated group, stacking, hydrophilicity, optoelectronic, catalytic and electrochemical properties of $\mathrm{rGO}$ strongly depends on reduction method. About various types of reduction techniques are discussed below.

\section{Thermal Reduction}

Directly GO is reduced by heating and this approach is called thermal reduction. In early stage of graphene synthesis, fast thermal annealing was applied to reduce and exfoliate simultaneously. ${ }^{26,27}$ The rapid heating generates $\mathrm{CO}$ or $\mathrm{CO}_{2}$ gas quickly. ${ }^{28}$ This rapid volume expansion makes layer exfoliation of graphene sheet. But, in this process wrinkled rGO sheet is obtained with wide size distribution as during decomposition of oxygenated group also carbon atoms are removed from the sheet. Heating condition considerably effects the degree of reduction. ${ }^{29}$ Generally with increase the temperature $\mathrm{C} / \mathrm{O}$ ratio increases for example at temperature less than $500^{\circ} \mathrm{C}, \mathrm{C} / \mathrm{O} \geq 7$, but temperature at $750^{\circ} \mathrm{C}$, the $\mathrm{C} / \mathrm{O}$ ratio $13 .{ }^{30}$ Like annealing temperature, annealing atmosphere plays important role on reduction of GO. Le et al., have demonstrated more clearly the role of $\mathrm{O}_{2}$ and $\mathrm{N}_{2}$ in thermal reduction of GO. Initially when the temperature relatively low $200^{\circ} \mathrm{C}$ both $\mathrm{O}_{2}$ and $\mathrm{N}_{2}$ atmosphere play similar role i.e. gradually loss of oxygen containing functional group. But temperature increases still $500^{\circ} \mathrm{C}$ in $\mathrm{O}_{2}$, makes more oxygen containing functional groups with defects 
in the GO surface compare to $\mathrm{N}_{2}$. As a result rGO shows less interlayer spacing in $\mathrm{O}_{2}$ compare to $\mathrm{N}_{2}$. Moreover, $\mathrm{O}_{2}$ can destroyed the carbon skeleton via ring rapturing of $\mathrm{GO}$ since $\mathrm{sp}^{3}$ carbon contain in $\mathrm{GO}$ is higher than $\mathrm{sp}^{2}$ carbon contain at $500^{\circ} \mathrm{C}$. In addition $\mathrm{GO}$ is most stable in $\mathrm{N}_{2}$ than $\mathrm{O}_{2}$ atmosphere that clearly observed in TGA result, GO completely combustion in $700^{\circ} \mathrm{C}$ in $\mathrm{O}_{2}$ but in $\mathrm{N}_{2} 20 \%$ remaining at $1000^{\circ} \mathrm{C} .{ }^{31}$ The annealing reduction mainly take place in vacuum ${ }^{32}$ or inert gas. ${ }^{33,34}$ Becerril et al., found that during thermal annealing of $\mathrm{GO}$ at $1100^{\circ} \mathrm{C}$ and vacuum pressure $<10^{-5}$ torr is necessary for the rGO production because the residual oxygen can be rapidly lost from the GO flim via reaction. ${ }^{32}$ This similar condition should be maintained in inert atmosphere. If the $\mathrm{H}_{2}$ is added in reaction chamber then prompt reaction happened with residual oxygen at higher temperature due to of high reduction capability of $\mathrm{H}_{2}$ at extremely higher temperatures. Thus reduction of $\mathrm{GO}$ can be performed in $\mathrm{H}_{2}$ atmosphere at comparatively lower temperature. Cheng et al., have described about the reduction of $\mathrm{GO}$ which take place at relatively lower temperature of $450^{\circ} \mathrm{C}$ for $2 \mathrm{~h}$ in the presence of equal mixture of $\mathrm{Ar} / \mathrm{H}_{2}$. The resultant $\mathrm{rGO}$ have greatly modified carbon and oxygen ratio of 14.9 and exhibited very high conductivity of $\sim 1000 \mathrm{~S} \mathrm{~cm}^{-1}{ }^{34}$ The presence of others stabilizing or protecting reagents GO can reduced by relatively lower temperature. Chen et al., proposed that $\mathrm{GO}$ was reduced at $150^{\circ} \mathrm{C}$ in $\mathrm{N}, \mathrm{N}$-dimethylacetamide/water under $\mathrm{N}_{2}$ atmosphere. Here dimethylacetamide acts as a protecting agent and alter the ratio of carbon and oxygen in GO to $\mathrm{rGO}$ from 2.09 to $4.70 .^{35} \mathrm{GO}$ was synthesized by simple conventional chemical exfoliation route so, during this reaction lot of oxygen incorporated in graphene skeleton due to the oxidation of ring carbon. Therefore we can called the GO as oxygen doped graphene and its possesses huge number of reactive sites which can easily react with others molecule in the suitable thermal condition. More recently many report published in doped graphene, this is nothing but replacement of oxygene in GO surface by others heteroatom such as nitrogen, boron, phosphorus, halogen, sulphur, selenium etc. This is actually chemically modifications but also possible in very facile way in surface modification by presence of some precursor molecules. Actually $\mathrm{GO}$ is the very important intermediate molecule for such transformation from graphite to others physical and chemically modified graphene. Dai group demonstrated about the simultaneous doping and reduction of GO molecules. According their report $\mathrm{GO}$ was heated at different temperature range from 300 to $1100^{\circ} \mathrm{C}$ in presence of $\mathrm{NH}_{3}$ as reactive gas and $\mathrm{Ar}$ as inert gas. The resultant product rGO contain maximum $5 \%$ nitrogen in carbon skeleton. ${ }^{36}$ There are several efforts to produce doped rGO. ${ }^{37}$

Till date have been reported that maximum 2D rGO prepared via thermal exfoliating from GO. The thermal exfoliation method could be used to generate random nanosheets with defects and also solubility of $\mathrm{rGO}$ is very limited in short range of solvent. However this exfoliation route is potentially most favorable for large-scale mass production method among the others exfoliation procedures. Moreover, overall exfoliation strategy is also highly useful and can be prolonged to the synthesis of rGO sheets.

\section{Microwave and Photo Reduction}

Microwave irradiation (MWI) is a current reliable approach for rGO synthesis due to reaction performed in very small time duration and with low power ingestion, In this process GO is reduced by $\mathrm{MWI}^{38,39}$ and photo treatment. ${ }^{40,41}$ The benefit of MWI than traditional thermal annealing approach is that it heat quickly and uniformly. The microwave treatment of $\mathrm{GO}$ in ambient condition produces $\mathrm{rGO}$ in just one minute..$^{38}$ In photo reduction GO is reduced by photo energy emitted by a flash lamp (xenon lamp). The flash energy provides 9 times higher energy than the energy required for reduction. So it causes sudden volume expansion which exfoliates the sheet eventually. As flash can simply exposed the rGO film and it is essential for fabricated pattern structure in electronic device. So, the photo eradiation is very helpful for various device fabrication of rGO since both reduction of $\mathrm{GO}$ and pattern formation happened simultaneously.

\section{Chemically Reduction}

Progresses of the physical exfoliation approaches of GO sheets have aided their rapid improvement. Still, it remains challenging to develop reduction technique that lead to the building of 2-D rGO with definite geometry and functional nanoarchitectures that are tailored to particular applications. The rGO can be synthesized at large scale by chemical reaction of $\mathrm{GO}$ with presence of 
reducing agent at room temperature or moderate temperature. Stankovich et al., first reduce the GO chemically by hydrazine. ${ }^{42}$ This report opens for facile approach of large scale synthesis of rGO. Many report showed that hydrazine is recommended as

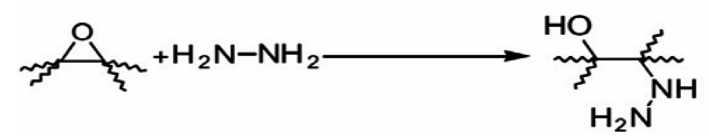

Fig. 4. Recommended the probable mechanism of epoxide ring opening in $\mathrm{GO}$ by $\mathrm{N}_{2} \mathrm{H}_{4}$ (ref. ${ }^{42}$ )

Like $\mathrm{N}_{2} \mathrm{H}_{4}, \mathrm{NaBH}_{4}$ acts as a valuable reducing agent for $\mathrm{GO}$ and it reduced $\mathrm{C}=\mathrm{O}$ to $\mathrm{O}-\mathrm{H}$ group but very poor efficiency for reduction of $-\mathrm{CO}_{2} \mathrm{H}$ and epoxy groups. ${ }^{44,45}$ So we can say that $\mathrm{NaBH}_{4}$ do not much hamper the solubility of rGO in wide range of solvent due to preserve its oxygen containing functional groups. ${ }^{46}$ Shin et al., found $\mathrm{NaBH}_{4}$ reduced $\mathrm{GO}$ has lower sheet resistance than $\mathrm{N}_{2} \mathrm{H}_{4}$ due to generate of $\mathrm{C}-\mathrm{N}$ bonds which made donors reimbursing the hole transfer in $\mathrm{rGO} .{ }^{47}$ The enhancement the degree of reduction of GO, Ajayan group ${ }^{48}$ have demonstrated an effective synthesis strategy of additional dehydration. According to their method $\mathrm{GO}$ was first treated with $\mathrm{NaBH}_{4}$ to cleavage the epoxy linkage after that product was treated with strong sulphuric acid at $180^{\circ} \mathrm{C}$ for removing the $-\mathrm{OH}$ groups. The final step is the high temperature annealing in inert atmosphere for completely eliminating the funtional group and recovering the $\mathrm{sp}^{2}$ network. The final product $\mathrm{rGO}$ contain carbon and oxygen ratio of 8.6 and its powder conductivity is $~ 16.6 \mathrm{~S} \mathrm{~cm}^{-1}$. Ascorbic acid can play important role of reducing agent of $\mathrm{GO}$, so it is great advantage compare to others reducing agents because its non-toxicity nature which is approving for treatment. Tasco'n co-workers demonstrated ascorbic acid as a good reducing agent of $\mathrm{GO}$ and which can be used in position of hydrazine. They showed that ascorbic acid reduced GO have contain carbon and oxygen ratio of $\sim 12.5$ with conductivity of $7700 \mathrm{~S} \mathrm{~m}^{-1}$ which is similar to those formed by $\mathrm{N}_{2} \mathrm{H}_{4}{ }^{49}$ Recently, Lee ${ }^{50}$ and Cheng group ${ }^{51}$ both individual reported a strong reducing agent of $\mathrm{HI}-\mathrm{AcOH}$ for reduction of $\mathrm{GO}$. The both results provide similar reduction aptitude that the resultant $\mathrm{rGO}$ possesses carbon and oxygen ratio of $\sim 15$ and the conductivity is $3.04 \times 10^{4} \mathrm{~S} / \mathrm{m}$. Recently Mandal et al., ${ }^{52}$ have reported a valuable synthesis method of $\mathrm{KI}$ reduction of $\mathrm{rGO}$ in acidic medium. $\mathrm{KI}$ is very mild oxidising agent compare to $\mathrm{HI}$ and there is no toxic effect so we can easily treated on GO. In this method they observed that total functional a valuable reagent for reduction of $\mathrm{GO}$ and it mainly transfer $\mathrm{C}=\mathrm{O}$ to $\mathrm{C}-\mathrm{H}$ group and new $\mathrm{C}-\mathrm{N}$ bond also formed in the graphite skeleton. ${ }^{42-45}$ The proposed reaction mechanism of reduction of epoxide group by hydrazine is shown below:

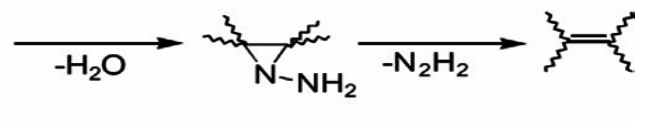

groups in initial precursor $\mathrm{GO}$ has almost intact after received the final product $\mathrm{rGO}$. The conductivity of $\mathrm{rGO}$ was $0.18 \mathrm{mS} \mathrm{cm}^{-1}$ is not too high compare others approach, but main problem is the cost of $\mathrm{KI}$ is expensive therefore large scale production for industrial manufacturing will be hindered.

\section{Photocatalytic Reduction}

In photocatalytic reduction GO is transfer to $\mathrm{rGO}$ by light with the support of photo-catalyst like titanium oxide. Recently, Kamat group ${ }^{53}$ demonstrated the GO reduction in a colloidal form by the help of titanium oxide nano-particle using irradiation of ultraviolet light source. The photocatalytic reduction of $\mathrm{GO}$ is clearly understood by observing colour change of GO solution gradually from light brown to black. The reaction start by charge separation in $\mathrm{TiO}_{2}$ particles upon UV-irradiation. The ethanol solvent present in reaction mixture scavenge the holes to generate $\mathrm{CH}_{3} \mathrm{CH}_{2} \mathrm{O}$ radicals, thus the exit electron is accumulated in the $\mathrm{TiO}_{2}$ nano-particles. These accrued electrons of $\mathrm{TiO}_{2}$ eventually reduce the GO. Besides $\mathrm{TiO}_{2}$, some other photocatalysts, like $\mathrm{ZnO}$ and $\mathrm{BiVO}_{4}{ }_{4}^{54}$ can play photocatalyst role for reduction of GO. Mangadlao et al. have introduced new photocatalyst of ketyl and it is effectively reduced GO in presence of 320-390 nm UV-light. The reduction experiment is clearly visualized by naked eye since after reduction $\mathrm{rGO}$ are precipitated due to loss of surface functional groups. The carbon and oxygen ratio of GO, GO in presence of UV-light, GO presence of both UV with catalyst were 2.6, 5.0 and 25.6, respectively. The significant increasing the carbon and oxygen ratio that imply the quickly eliminating of oxygen-containing funtional group by ketal radical as catalyst..$^{55}$ Hou et al., observed that sunlight acts as a reducing as well as cutting tool of GO. The experiment have exhibited that initially reduction rate of $\mathrm{GO}$ is rapid and not sensitive to the dissolved oxygen amount but after long time reaction depend on the dissolved oxygen. ${ }^{56}$ 


\section{Electrochemical Reduction}

GO has numerous number of oxygen containing redox active functional groups, thus electrochemical approach can be fabricated the rGO molecule via redox reaction. In this technique after coated a skinny film of GO on an electrode (such as FTO or ITO coated glass), a negative potential is applied on it in three electrode configuration to reduce it, where buffer solution is used as electrolyte. Here the reduction mechanism involves mainly electron transfer from electrode substrate to GO. Ramesha and Sampth synthesized rGO by cyclic voltammetry was performed in $0.1 \mathrm{M}$ potassium nitrate at $10 \mathrm{mV} / \mathrm{s}$ in the potential range 0 to $-1 \mathrm{~V}$ vs $\mathrm{Hg}, \mathrm{Hg}_{2} \mathrm{Cl}_{2} / \mathrm{Cl}^{-}$saturated. They observed that the reduction initiate at $-0.6 \mathrm{~V}$ and extents to maximum at $-0.87 \mathrm{~V}$. The formation of rGO clearly detected from their Raman spectra i.e. G band of GO at 1610 $\mathrm{cm}^{-1}$ was shifted to $1585 \mathrm{~cm}^{-1}$ of rGO ${ }^{57}$ Pereira group synthesized rGO by electrochemical treatment on $\mathrm{GO}$ in phosphate buffer solution in the potential of $-0.8 \mathrm{~V}$ for different times interval. The fabricated $\mathrm{rGO}$ was characterized by in-situ atomic force microscopy and Raman scattering and result showed that $I_{D} / I_{G}$ ratio in Raman spectra increases with reaction time due to more reduction of GO sheet. ${ }^{58}$ There are several attempts to electrochemically reduce GO. ${ }^{59-61}$ The merit of this reduction technique is that there is no necessity to use of any hazardous reagent and the rGO film can be directly applied in different application purpose.

\section{Solvothermal Reduction}

Reduced graphene can be produced by diverse chemical and physical techniques, but all these methods have their advantages and disadvantage in the point of temperature, production, chemical structure, cost and purity. Scientist has provided a valuable mild synthesis conditions for reduction of GO with large mass production in presence of some solvent, it is called solvothermal/ hydrothermal. Generally solvothermal/hydrothermal process is implemented in a closed container under heating condition. In this process solvent play an important role to increase the pressure inside the container as a result temperature reach above the boiling point and consequently thermal energy increases. Dai group have demonstrated the synthesis of rGO by solvothermal method using solvent $\mathrm{N}, \mathrm{N}$-dimethylformamide. ${ }^{62}$ In this process very low quantity of $\mathrm{N}_{2} \mathrm{H}_{4}$ was needed for reduction and the solvothermal treatment is performed at $180^{\circ} \mathrm{C}$ for half day. The resultant rGO shows high carbon contain (C/O ratio 14.3) compare to rGO produced by $\mathrm{N}_{2} \mathrm{H}_{4}$ reduction at ambient condition. Dubin et al., have synthesised rGO from 1-methyl-2pyrrolidinone (NMP) dispersed GO by solvothermally. Here NMP not only acts as high boiling solvent but also used as oxygen scavenger which help to deoxygenated the GO sheet. The rGO contains carbon and oxygen ratio of 5.15 where GO has carbon and oxygen of 2.34 and conductivity of rGO sheet was $3.74 \times 10^{2} \mathrm{~S} / \mathrm{m} .{ }^{63}$ Loh co-workers have reduced GO in autoclave at $180^{\circ} \mathrm{C}$ for 6 hours. ${ }^{64}$ In this approach neutral $\mathrm{H}_{2} \mathrm{O}$ used as a reducing agents because at high temperature water liberate high amount $\mathrm{H}^{+}$ions which promote the catalytic reaction of $\mathrm{GO}$. The reduction of $\mathrm{GO}$ take place via intramolecular or intermolecular process i.e. formation of $\mathrm{H}_{2} \mathrm{O}$ molecule or ether linkage. Chen et al., published a similar paper but difference is the sample did not contact directly with the solvent only reaction take place in solid/gas interface in hydrothermal condition. In this synthesis both water and ammonia solvent are used separately for reduction of free standing GO paper and the final products rGO showed high conductivity of $900 \mathrm{~S} / \mathrm{m}$ and $2283 \mathrm{~S} / \mathrm{m}$ respectively. ${ }^{65}$

The hydothermal/solvothermal is a facile, efficient, and inexpensive approach for the fabrication of $\mathrm{rGO}$ with high production. And also most important factor is the hydrothermally synthesized rGO have good solubility in the wide range of solvent compare to others approaches due to almost preserve its functional groups after treatment. But, the synthesis is highly depend to the experimental circumstances. Any changes in the type capping agents or amount, solvent, reaction time and temperature would result in vast changes in the final products.

\section{GO and rGO based Supercapacitor}

Among different energy storage device, supercapacitor is a superior due capable to store high energy density. Fig. 5 depicts the Ragone plot of supercapacitors comparing with other energy storage systems and it clearly evidences that supercapacitor has high prospect of reaching at battery like energy density with its ultrahigh power density.

2-D GO and rGO materials are extensively used as electrode of supercapacitor because of their 
following advantages: Their 2-D structure provides large effective surface area which enhances EDLC type capacitance. Presence of their intrinsically rich negative polarisable/ionisable oxygen prefers Faradic charge storage performance (pseudocapacitive). 2-D structure reduces the ion diffusion length during charging which makes their charge storage nature capacitive. This is the fundamental property of an electrode of supercapacitor. Their high mechanical strength and flexibility help to fabricate flexible and portable supercapacitors.

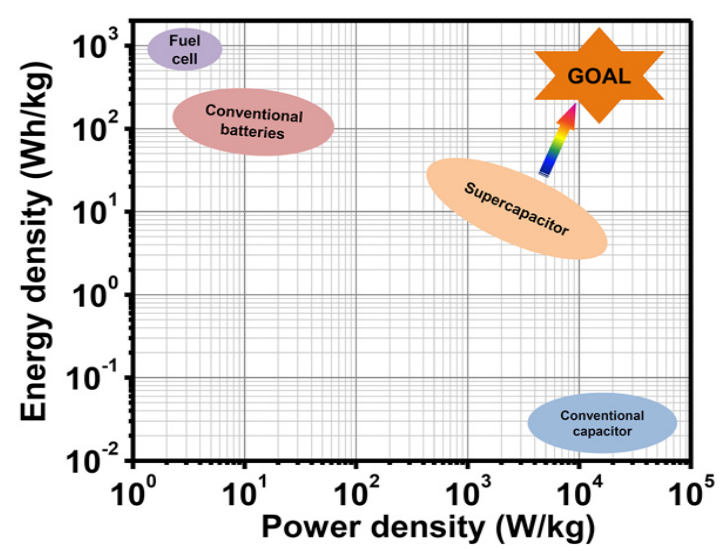

Fig. 5. Ragone plot of different energy storage systems

Reduced graphene oxide has attracted extensive interest in the field of supercapacitor research because of its 2-D structure which bequests it exceptional properties such as large effective surface area of $2630 \mathrm{~m}^{2} \mathrm{~g}^{-1}$, superior electrical conductivity and mechanical properties. rGO not only shows exceptional EDLC capacitance via access of electrolyte to its both surfaces, but also it exhibits good pseudocapacitance nature via its redox active oxygenated groups $(>\mathrm{C}=\mathrm{O},-\mathrm{COOH}$, $-\mathrm{OH}$, etc.). Stoller et al., first discovered graphenebased EDLCs using chemically modified graphene (CMG, synthesized via reduction of $\mathrm{GO}$ by hydrazine hydrate) as electrode materials. ${ }^{66}$ Though this rGO sheets was agglomerated into particles, but it shows of $705 \mathrm{~m}^{2} / \mathrm{g}$ surface area. This CMG exhibits capacitance of 99 and $135 \mathrm{~F} / \mathrm{g}$ in organic and aqueous electrolytes, respectively. Afterwards there were various strategies to improve the capacitance of rGO. Wang et al., applied gas-solid reduction method on $\mathrm{GO}$ precursor by $\mathrm{N}_{2} \mathrm{H}_{4}$ for minimizing the agglomeration of $\mathrm{rGO} .{ }^{67}$ This $\mathrm{rGO}$ shows maximum charge storage of $205 \mathrm{~F} / \mathrm{g}$ and an energy capacity of $28.5 \mathrm{~W} \mathrm{~h} / \mathrm{kg}$ with power capacity of $10 \mathrm{~kW} / \mathrm{kg}$. Chen et al., ${ }^{68}$ measured supercasitor property for mildly reduced $\mathrm{GO}$ and exhibits high capacitance of $348 \mathrm{~F} / \mathrm{g}$ at $0.2 \mathrm{~A} / \mathrm{g}$ due to presence of maximum redox active oxygenated groups in rGO surface. Recently Mandal et al., ${ }^{52}$ reported KI reduced rGO for supercapacitor application. This rGO exhibited specific capacitance of $414 \mathrm{~F} / \mathrm{g}$ at $0.5 \mathrm{~A} \mathrm{~g} \mathrm{~g}^{-1}$ with high operation stability 95\% after 10,000 cycles. Also It has high rate capability of $56.5 \%$ at $20 \mathrm{~A} \mathrm{~g}^{-1}$ and energy density of $10.76 \mathrm{~W} \mathrm{~h} / \mathrm{kg}$ with power density of $125 \mathrm{~W} \mathrm{~kg}^{-1}$. The high performance of rGO due to the presence of enormous number of redox active oxygen containing functional groups which enhance the capacitace value of rGO through Faradic reaction. The application of rGO as electrode materials is not only limited upto here; again its application has been extended by decorating conducting polymers and different active nanoparticles on it and the synthesized composite materials show exceptional charge storage performance. ${ }^{69}$

Table 1: Comparison of capacitive performance of differently synthesized rGO

\begin{tabular}{lccc}
\hline Electrode Material & Capacitance( F/g) & Experiment Parameter (A/g) & References \\
\hline Thermally reduced rGO & 260.5 & 0.4 & 70 \\
Hydrothemally reduced rGO & 367 & 1 & 71 \\
Solvothermally reduced rGO & 276 & 0.1 & 72 \\
HBr reduced rGO & 348 & 0.2 & 73 \\
Thermally reduced rGO & 41 & 1 & 74 \\
KI reduced rGO & 414 & 0.5 & 52 \\
GO (modified Hummers's method) & 189 & 0.05 & 75 \\
Thermally reduced rGO & 281 & 1 & 76 \\
Hydrothemally reduced rGO & 308 & 3 & 77 \\
Thermally reduced rGO & 85.3 & 1 & 78 \\
Thermally reduced rGO & 381 & 1 & 79 \\
Hydrothemally reduced rGO & 326 & 0.2 & 80 \\
electrochemical reduction rGO & 158.5 & 0.5 & 81 \\
\hline
\end{tabular}




\section{CONCLUSION}

Strong characteristic features of 2-D GO and rGO are very helpful for application in different fields. In that prospect, synthesis of $\mathrm{GO}$ and $\mathrm{rGO}$ materials is a spirited job. In case of rGO, through reduction of $\mathrm{GO}$ is common method for its synthesis; but the nature of the synthesized rGO such as degree of reduction, stacking of rGO sheets, retention of redox active oxygenated groups is strongly depends on reduction technique. In this short review various method are highlighted in the point of reaction time, production, solvent, functional group, surface area temperature, etc. Regarding future prospect of rGO in different device fabrication, therefore enormous number of work is going on and researcher are still searching a new synthesis strategy for accurately regulate the reduction of GO. However, research on rGO upto today is still far away from saturation due to uncontrolled materials obtained from the current synthesis methods. Still lot of synthesis approach are available in market such as physical and chemical vapor deposition technique, arc discharge, etc. but all these have lots of limitation in the point of solubility, product yield, reaction condition. The emerging applications of GO and rGO materials for supercapacitor are clearly mentioned in this review article.

\section{ACKNOWLEDGEMENT}

The author acknowledges Dr. Debasish Mandal, Post-doctoral fellow at Chemical and Biomoleular Enginnering, Yonsei University, South Korea for his help and cooperation.

\section{Conflicts of Interest}

The author declare no conflict of interest.

\section{REFERENCES}

1. Velicky, M.; Tothc, S. P. Applied Materials Today., 2017, 8, 68-103.

2. Kumar, S. K.; Choudhary, N.; Jung, Y.; Thomas, J. ACS Energy Lett., 2018, 3, 482-495.

3. Chhowalla, M.; Liu, Z.; Zhang, H. Chem. Soc. Rev., 2015, 44, 2584-2586.

4. Mandal, D.; Routh, P.; Nandi, K.A. Small., 2018, 14, 1702881- 1702881.

5. Mandal, D.; Routh, P.; Nandi, K. A. Chem. Asian J., 2018, 13, 3871-3884.

6. Geng, D.; Yang, H. Y. Adv. Mater., 2018, 30, 1800865-1800865.

7. Zeng, M.; Xiao, Y.; Liu, J.; Yang, K.; Fu L. Chem. Rev., 2018, 118, 6236-6296.

8. Lin, Y.; Connell, J. W. Nanoscale., 2012, 4, 6908-6939.

9. Zhu, Y.; Murali, S.; Cai, W.; Li, X.; Suk, J.W.; Potts, J. R.; Ruoff, R. S. Adv. Mater., 2010, 22, 3906-3924.

10. Singh, R. K.; Kumar, R.; Singh, D. P. RSCAdv., 2016, 6, 64993-65011.

11. Loh, K. P.; Bao, Q.; Eda, G.; Chhowalla, M. Nature Chemistry., 2010, 2, 1015-1024.

12. Li, F.; Jiang, X.; Zhao, J.; Zhang, S. Nano Energy., 2015, 16, 488-515.

13. Tarcan, R.; Todor-Boer, O.; Petrovai, I.; Leordean, C.; Astilean, S.; Botiz, I. J. Mater. Chem. C., 2020, 8, 1198-1224.

14. Tadyszak, K.;Wychowaniec, J.K.; Litowczenko, J. Nanomaterials., 2018, 8, 944-964.
15. Xiaosheng, C.; Qilu, Z.; Shuojue; W.; Jing, P.; Youwei, Z.; Huiling, M.; Jiuqiang, L.; Maolin, Z. Journal of Materials Science., 2014, 49, 5667-5675.

16. Yan, Y.; Gu, P.;Zheng, S.;Zheng, M.;Pang, H.; Xue, H. J. Mater. Chem. A., 2016, 4, 19078-19085.

17. Simon, P.; Gogotsi Y.; Dunn, B. Science., 2014, 343, 1210-1211.

18. Kuila, T.; Mishra A. K.; Khanra, P.; Kim, N. H.; Uddin, M. E.; Lee, H. J. Langmuir., 2012, 28, 9825-9833.

19. Xu, B.; Yue, S.; Sui, Z.; Zhang, X.; Hou, S.; Cao, G.; Yang, Y. Energy Environ. Sci., 2011, 4, 2826-2830.

20. Rowley-Nealea, S. J.; Randviir, E. P.; Denac, A. S. A.; Banks, C. E. Applied Materials Today., 2018, 10, 218-226.

21. Chua, C. K.; Pumera, M. Chem. Soc. Rev., 2014, 43, 291-312. (b) Swain, S. S.; Unnikrishnan, L.; Mohanty, S.; Nayak, S. K. J. Mater. Sci., 2018, 53, 15442-15464.

22. Brodie, B. C. Ann. Chim. Phys., 1855, 45, 351.

23. Hofmann, U.; Frenzel, A. Ber. dtsch. Chem. Ges., 1930, 898, 1248- 1262.

24. Hummers, S. W.; Offeman, E. R. J. Am. Chem. Soc., 1958, 80, 1339.

25. Marcano, D. C.; Kosynkin, D. V.; Berlin, J. M.; Sinitskii, A.; Sun, Z.; Slesarev, A.; Alemany, L. B.; Lu, W.; Tour, J. M. ACS Nano., 2010, 4, 4806-4814. 
26. Wu, Z. S.; Ren, W.; Gao, L.; Liu, B.; Jiang, C.; Cheng, H. M. Carbon., 2009, 47, 493-499.

27. Schniepp, C. H.; Li, L. J.; McAllister, J. M.; Sai, H; Herrera, M. A.; Adamson, D. H.; Prud'homme, R. K.; Car, R.; Saville, D. A.; Aksay, I. A. J Phys Chem B., 2006, 110, 8535- 8539.

28. Wang, Z.-I.; Xu, D.; Huang, Y.; Wu, Z.; W. L.-m.; Zhang, X.-b. Chem. Commun., 2012, 48, 976-978.

29. Shang, Y.; Zhang, D.; Liu, Y.; Guo, C.; Bull. Mater. Sci., 2015, 38, 7-12.

30. Pei, S.; Cheng, H.-M. Carbon., 2012, 50, 3210-3228.

31. Le, G. T. T.; Manyam, J.; Opaprakasit, P.; Chanlek, N.; Grisdanurak, N.; Sreearunothai, P.; Diamond \& Related Materials., 2018, 89, 246-256.

32. Becerril, H. A.; Mao, J.; Liu, Z.; Stoltenberg, R. M.; Bao, Z.; Chen, Y. ACS Nano., 2008, 2, 463-470.

33. Wang X.; Zhi, L.; Mullen, K. Nano Lett., 2008, 8, 323-327.

34. Wu, Z-S.; Ren, W.; Gao, L.; Zhao, J.; Chen, Z.; Liu, B.; Tang, D.; Yu, B.; Jiang, C.; Cheng, H.-M. ACS Nano., 2009, 3, 411-417.

35. Chen, W.; Yan, L. Nanoscale., 2010, 2, 559-563.

36. Li, X.; Wang, H.; Robinson, J. T.; Sanchez, H.; Diankov, G.; Dai, H. J. Am. Chem. Soc., 2009, 131, 15939- 15944.

37. Wang, X.; Sun, G.; Routh, P.; Kim, D.-H.; Huang, W.; Chen, P. Chem. Soc. Rev., 2014, 43, 7067-7098.

38. Zhu, Y.; Murali, S.; Stoller, M. D.; Velamakanni, A.; Piner, R. D.; Ruoff, R. S. Carbon., 2010, 48, 2118- 2122.

39. Hassan, A. H. M.; Abdelsayed, V.; Khder, R. S. A. E.; AbouZeid, K. M.; Terner, J.; El-Shall, M. S. J. Mater. Chem., 2009, 19, 3832- 3837.

40. Cote, L. J.; Cruz-Silva, R.; Huang, J. J. Am. Chem. Soc., 2009, 131, 11027-11032.

41. Zhang, Y.; Guo, L.; Wei, S.; He, Y.; Xia, H.; Chen, Q. Nanotoday., 2010, 5, 15-20.

42. Stankovich, S.; Dikin, D. A.; Piner, R. D.; Kohlhaas, K. A.; Kleinhammes, A.; Jia, Y.; Wu, Y.; Nguyen, S. T.; Ruoff, R. S. Carbon., 2007, 45, 1558-1565.

43. Gomez-Navarro, C.; Weitz, T. R.; Bittner, M. A.; Scolari, M.; Mews, A.; Burghard, M.; Kern, K. Nano Lett., 2007, 7, 3499-3503.
44. Mattevi, C.; Eda, G.; Agnoli, S.; Miller, S.; Mkhoyan, K. A.; Celik, O.; Mastrogiovanni, D.; Granozzi,G.; Garfunkel, E.; Chhowalla, M. Adv. Funct. Mater., 2009, 19, 2577-2583.

45. Periasamy, M.; Thirumalaikumar, M. J Organo Metallic Chem., 2000, 609, 137-151.

46. Li, D.; Muller, M. B.; Gilje, S.; Kaner, R. B.; Wallace, G. G. Nat. Nanotechnol., 2008, 3, 101-105.

47. Shin, H-J.; Kim, K. K.; Benayad, A.; Yoon, S-M.; Park, H. K.; Jung, I.-S. Jin, H. M.; Jeong, H.-K.; Kim, J. M.; Choi, J.-Y.; Lee, H. Y. Adv Funct Mater., 2009, 19, 1987-1992.

48. Gao, W.; Alemany, L. B.; Ci, L.; Ajayan, P. M. Nat Chem., 2009, 1, 403-408.

49. Fernandez-Merino, J. M.; Guardia, L.; Paredes, I. J.; Villar-Rodil, S.; Solis-Fernandez, P.; Martinez-Alonso, A.; Tasco'n, J. M. D. J Phys Chem C., 2010, 114, 6426-6432.

50. Moon, K. I; Lee, J.; Ruoff, R. S.; Lee, H. Nat Comm., 2010, 1, 1-6.

51. Pei, S.; Zhao, J.; Du, J.; Ren, W.; Cheng, H-M. Carbon., 2010, 48, 4466-4474.

52. Mandal, D.; Routh, P.; Nandi, A. K. Chemistry Select., 2017, 2, $3163-3171$.

53. Williams, G.; Seger, B.; Kamat, P. V. ACS Nano., 2008, 2, 1487-1491.

54. Williams, G.; Kamat, P. V. Langmuir., 2009, 25, 13869-13873.

55. Mangadlao, J.; Choi, D.; Cao, P.-F.; Advincula, R. C. ACS Appl. Mater. Interfaces., 2017, 9, 24887-24898.

56. Hou, W.-C.; Chowdhury, I.; Goodwin, D. G.; Henderson, J. W. M.; Fairbrother, D. H.; Bouchard, D.; Zepp R. G. Environ. Sci. Technol., 2015, 49, 3435-3443.

57. Ramesha, G. K.; Sampath, S. J. Phys. Chem., C, 2009, 113, 7985-7989.

58. Moraes, F. C.; Freitas, R. G.; Pereira, R.; Gorup, L. F.; Cuesta, A.; Pereira, E. C. Carbon., 2015, 91, 11-19.

59. Zhou, M.;Wang, Y.; Zhai, Y.; Zhai, J.; Ren, W.; Wang, F.; Dong, S. Chem. Euro. J., 2009, 15, 6116- 6120.

60. Wang, Z.; Zhou, X.; Zhang, J.; Boey, F.; Zhang, H. J. Phys. Chem. C., 2009, 113, 14071- 14075.

61. An, S. J.; Zhu, Y.; Lee, S. H.; Stoller, M. D.; Emilsson, T.; Park, S.; Velamakanni, A.; An J.; Ruoff, S. R. J. Phys. Chem. Lett., 2010, 1, 1259-1263. 
62. Wang, H.; Robinson, J. T.; Li, X.; Dai, H. J. Am. Chem. Soc., 2009, 131, 9910-9911.

63. Dubin, S.; Gilje, S.; Wang, K.; Tung, V. C.; Cha, K.; Hall, A. S.; Farrar, J.; Varshneya, R.; Yang, Y.; Kaner, R. B. ACS Nano., 2010, 4, 3845-3852.

64. Zhou, Y.; Bao, Q.; Tang, L. A. L.; Zhong, Y.; Loh, K. P. Chem. Mater., 2009, 21, 2950- 2956.

65. Chen, H., Song, Z.; Zhao, X.; Li, X.; Lin H. RSC Adv., 2013, 3, 2971-2978.

66. Stoller, M. D.; Park, S.; Zhu, Y.; An, J.; Ruoff, R. S. Nano Lett., 2008, 8, 3498-3502.

67. Wang, Y.; Shi, Z.; Huang, Y.; Ma, Y.; Wang, C.; Chen, M.; Chen, Y. J. Phys. Chem. C., 2009, 113, 13103- 13107.

68. Chen, Y.; Zhang, X.; Zhang, D.; Yu, P.; Ma, Y. Carbon., 2011, 49, 573-580.

69. Tan, Y. B.; Lee, J-M. J. Mater. Chem. A., 2013, 1, 14814-14843.

70. Zhao, B.; Liu, P.; Jiang, Y.; Pan, D.; Tao, H.; Song, J.; Fang, T.; Xu, W. J. Power Source., 2012, 198, 423-427.

71. Johra. F. T.; Jung, W.-G. Appl. Surf. Sci., 2015, 357, 1911-1914.

72. Lin, Z.; Liu, Y.; Yao, Y.; Hildreth, O. J.; Li, Z.; Moon, K.; Wong, C. J. Phys. Chem. C., 2011,
115, 7120-7125.

73. Chen, Y.; Zhang, X.; Zhang, D.; Yu, P.; Ma, Y. Carbon., 2011, 49, 573-580.

74. Yang, J.; Gunasekaran, S. Carbon., 2013, 51, 36-44.

75. Xu, B.; Yue, S.; Sui, Z.; Zhang, X.; Hou, S.; Cao G.; Yang, Y. Energy Environ. Sci., 2011, 4, 2826-2830.

76. Zuo, Z.; Jiang, Z.; Manthiram, A. 60. J. Mater. Chem. A., 2013, 1, 13476-13483.

77. Guo, H. L.; Su, P.; Kang, X. F.; Ning, S. K. J. Mater. Chem. A., 2013, 1, 2248-2255.

78. Nam, P.T.; Khanh, N. V.; Thom, N. T.; Phuong, N. T.; Trang, N. V.; Xuyen, N. T.; Thai, V. Q.; Tuan, V. A.; Thanh, D. T. M.; Vietnam J. Chem., 2018, 56, 778-785.

79. Fan, W.; Xia, Y.-Y.; Tjiu, W. W.; Pallathadka, P. K.; He, C.; Liu, T. J. Power Sources., 2013, 243, 973-981.

80. Sun, L.; Wang, L.; Tian, C.; Tan, T.; Xie, Y.; Shi, K.; Li, M.; Fu, H. RSC Adv., 2012, 2, 4498-4506.

81. Tong, H.; Zhu, J.; Chen, J.; Han, Y.; Yang, S.; Ding, B.; Zhang, X. J Solid State Electrochem., 2013, 17, 2857-2863. 